\title{
Avaliação dos eventos adversos relacionados ao procedimento cirúrgico no ambiente hospitalar: uma revisão na literatura
}

\begin{abstract}
RESUMO | Objetivou-se analisar os eventos adversos mais frequentes ao paciente cirúrgico e identificar os principais fatores associados. Trata-se de uma revisão sistemática a partir da pergunta norteadora "Quais eventos adversos ocorrem com maior frequência no centro cirúrgico?". Foram incluídos estudos baseados na avaliação de eventos adversos e complicações cirúrgicas publicados no período de 2007 a 2017. A Busca atingiu 2035 publicações. Após aplicação dos critérios foram selecionados 13 artigos. Principais eventos encontrados: erros de lateralidade, punção/laceração acidental, corpo estranho deixado durante o procedimento, complicações cirúrgicas, suspensão de cirurgia, alterações respiratórias/instabilidade hemodinâmica, problema relacionado ao sítio cirúrgico, obstrução intestinal, hemorragia ou hematoma pós-operatório, reação a transfusão, parada/falha respiratória, falha na reanimação e falha na avaliação pré-operatória. A partir deste estudo os profissionais podem rever os processos e seus fluxos no bloco cirúrgico a fim de desenvolver ou fortalecer barreiras para reduzir os danos e contribuir para segurança do paciente.
\end{abstract}

Palavras-chaves: Centro cirúrgico, Enfermagem Perioperatória, Segurança do paciente, Serviço hospitalar de enfermagem.

ABSTRACT | The objective was to analyze the most frequent adverse events in the surgical patient and to identify the main associated factors. This is a systematic review based on the guiding question "Which adverse events occur most frequently in the operating room?". We included studies based on the assessment of adverse events and surgical complications published from 2007 to 2017. The Search reached 2035 publications. After applying the criteria, 13 articles were selected. Main events found: laterality errors, accidental puncture / laceration, foreign body left during the procedure, surgical complications, surgery suspension, respiratory changes / hemodynamic instability, surgical site-related problem, intestinal obstruction, postoperative bleeding or hematoma, reaction transfusion, respiratory arrest / failure, resuscitation failure, and preoperative assessment failure. From this study, professionals can review the processes and their flows in the operating room in order to develop or strengthen barriers to reduce damage and contribute to patient safety.

Keywords: Surgicenters, Perioperative Nursing, Patient safety, Nursing hospital service.

RESUMEN | El objetivo fue analizar los eventos adversos más frecuentes en el paciente quirúrgico e identificar los principales factores asociados. Esta es una revisión sistemática basada en la pregunta guía "¿Qué eventos adversos ocurren con mayor frecuencia en el quirófano?". Se incluyeron estudios basados en la evaluación de eventos adversos y complicaciones quirúrgicas publicados entre 2007 y 2017. La búsqueda llegó a 2035 publicaciones. Después de aplicar los criterios, se seleccionaron 13 artículos. Principales eventos encontrados: errores de lateralidad, punción / laceración accidental, cuerpo extraño dejado durante el procedimiento, complicaciones quirúrgicas, suspensión quirúrgica, cambios respiratorios / inestabilidad hemodinámica, problema relacionado con el sitio quirúrgico, obstrucción intestinal, sangrado postoperatorio o hematoma, reacción transfusión, paro / falla respiratoria, falla de reanimación y falla de evaluación preoperatoria. A partir de este estudio, los profesionales pueden revisar los procesos y sus flujos en el quirófano para desarrollar o fortalecer barreras para reducir daños y contribuir a la seguridad del paciente.

Palabras claves: Centros Quirúrgicos, Enfermería Perioperatoria, Seguridad del paciente Servicio de enfermería hospitalaria.

Juliane Rocha de Oliveira

Bacharela em enfermagem pela Universidade Federal do Espírito Santo.

\section{Samuel Goncalves Rodrigues}

Bacharel em enfermagem pela Universidade Federal do Espírito Santo.

\section{Kenia Pimentel Rangel \\ Enfermeira, Mestre em Gestão Pública- UFES \\ Paula de Souza Silva Freitas}

Enfermeira, Mestre e Doutora em Saúde Coletiva, professora adjunta do curso de enfermagem da UFES.

\section{Rita Simone Lopes Moreira}

Enfermeira, Mestre em Enfermagem. Doutora em Cardiologia, Professora Adjunta do curso de Enfermagem da UNIFESP, coordenadora da Residência Multiprofissional em cardiologia da UNIFESP.

\section{Bruno Henrique Fiorin}

Enfermeiro, Mestre em Saúde Coletiva (UFES). Doutor em Cardiologia (UNIFESP), Professor Adjunto do curso de enfermagem da UFES e colaborador da Pós Graduação em gestão Pública da UFES.

Recebido em: 15/07/2019

Aprovado em: 18/09/2019
INTRODUÇÃO

A temática "segurança do paciente" tem sido considerada prioridade nas questões que dizem respeito à gestão em saúde, visto que é uma dimensão da qualidade e um desafio nas organizações. Diversos esforços têm sido realizados para poder conscientizar os profissionais de que é necessário ampliar a cultura de segurança em suas instituições. Há quase duas décadas a temática relacionada a eventos adversos se tornou objeto de estudo no mundo ${ }^{1}$.Estima-se que $4 \%$ a $16 \%$ dos pacientes hospitalizados serão 
acometidos com algum tipo de evento adverso. É fato que se trata de um problema de saúde pública de importância internacional; sendo crescente o envolvimento de pesquisadores, profissionais de saúde e gestores de saúde na verificação destas ocorrências ${ }^{2,3}$.

A atenção à saúde no nível terciário vem atendendo um volume cada vez maior de indivíduos críticos e com maior grau de complexidade. Nesta situação, os clientes estão mais vulneráveis aos riscos oriundos dessa assistência, como infecção, erros por parte dos profissionais, incidentes ou eventos sentinelas. Estudos têm concluído que eventos adversos alcançam maiores proporções em setores em que os procedimentos requerem um alto nível de complexidade ${ }^{4,5}$.

Nas instituições hospitalares a complexidade e a tecnologia na assistência à saúde estão relacionadas as altas taxas de eventos adversos em unidades de terapia intensiva, as salas de emergência e as salas operatórias, mais especificamente em cirurgia vascular, cirurgia cardíaca e neurocirurgia ${ }^{6}$. Com o relatório To Erris Human: Building a Safer Health System publicado em 2000, os eventos adversos se tornaram mais evidentes, trazendo à tona a necessidade de as instituições de saúde garantirem a segurança ao paciente. Este relatório aponta que mais de dois terços dos eventos adversos eram erros evitáveis, e apontou ainda meios de intervir, enfatizando os mecanismos de vigilância, denúncia ou inspeção para identificação dos eventos e implementação de medidas corretivas ${ }^{6,7}$.

Evento Adverso é uma ocorrência não intencional que gera ou que pode gerar consequências ao paciente. Já o incidente é entendido como evento ou circunstância que poderia ter gerado consequências ou que resultou de forma evitável ${ }^{8,9}$. Um evento adverso evitável é um erro que resulta em dano decorrente da intervenção de um profissional desqualificado, negligente ou imprudente ${ }^{6,10}$.

O termo Segurança do paciente aplica-se as iniciativas para evitar, prevenir e reduzir resultados adversos ocorridos a partir do cuidado à saúde ${ }^{11}$.Diante das evidências, a partir de 2004, a ANVISA (Agência Nacional de Vigilância em Saúde) inseriu ao seu escopo de atuação, ações previstas pela OMS (Organização Mundial da Saúde) na World Alliance for Patient Safety, e em 2013 lançou por meio do Protocolo de Segurança Cirúrgica a lista de verificação de segurança cirúrgica que configura uma estratégia importante para a consolidação de práticas mais seguras na realização de procedimentos cirúrgicos, no local correto e no paciente correto, reduzindo os eventos adversos, os riscos de incidentes e a mortalidade cirúrgica ${ }^{4,12}$.

No ano de 2008 foram realizadas 234 milhões de cirurgias no mundo, equivalendo a uma para cada 25 pessoas vivas. Morreram dois milhões de pacientes nesses procedimentos e cerca de sete milhões apresentaram complicações, sendo que $50 \%$ dessas complicações foram consideradas evitáveis. De cada 300 pacientes admitidos nos hospitais morre um paciente, mais de $50 \%$ desses pacientes são cirúrgicos e com eventos preveníeis. Esses são dados inadmissíveis para o século XXI, o que levou a OMS e a Universidade Harvard a iniciar uma campanha mundial "Cirurgia Segura Salva Vidas" para reduzir estes indicadores ${ }^{3,13}$.

Um estudo realizado no Rio de Janeiro sobre as características de eventos adversos observou que a ocorrência de 30,8\% ocorre no Centro Cirúrgico, o mesmo estudo buscou os fatores contribuintes e identificou como principal motivo a não adesão as normas institucionais. A mediana de incidência global de eventos adversos intra-hospitalares é de 9,6\%, quase um a cada 10 pacientes, com percentual médio de $43,5 \%$ de evitabilidade, sendo $7,4 \%$ de eventos letais e $56,3 \%$ de incapacidades leves ou graves ${ }^{7,10}$.

No período de 2006-2009, a World Alliance for Patient Safety deu início ao segundo desafio global, e estabeleceu o foco na melhoria da segurança no ambiente cirúrgico (Cirurgia Segura), por meio de quatro ações importantes: prevenção de infecções do sítio cirúrgico; anestesia segura; equipes cirúrgicas seguras e indicadores da assistência cirúrgica ${ }^{3}$.

Nesse sentido, identificar os eventos adversos mais frequentes no centro cirúrgico, disponíveis na literatura, podem fornecer subsídios para reorganização do setor, bem como maior segurança no futuro. Julgou-se relevante desenvolver essa revisão sistemática. Desta forma, objetivou-se analisar os eventos adversos mais frequentes relacionados ao paciente cirúrgico e identificar na literatura os principais fatores associados como o intuito de responder a inquietação de quais são os eventos adversos mais comuns no ambiente cirúrgico.

\section{MÉTODO}

Trata-se de uma revisão sistemática realizada entre os meses de dezembro de 2018 a fevereiro de 2019, a fim de responder a seguinte pergunta norteadora: Quais eventos adversos ocorrem com maior frequência no centro cirúrgico? Para levantamento de dados, a busca foi realizada nas bases de dados Scientific Electronic Library Online (SciELO), Literatura Latino-americana e do Caribe em Ciências da Saúde (LILACS), Base de Dados de Enfermagem (BDENF) e Literatura Internacional em Ciências da Saúde (MEDLINE). Os descritores empregados para a seleção dos estudos foram escolhidos com base nos Descritores em Ciências da Saúde (DeCS) e Medical Subject Headings (Mesh terms), e em outros termos relevantes sobre a temática, sendo utilizados os seguintes descritores: Centro Cirúrgico, Gestão de Risco, Medidas de Segurança, Segurança do Paciente e, o termo associado, Eventos Adversos.

Foram definidos como critérios de inclusão, estudos baseados na avaliação de eventos adversos (EAs) e complicações cirúrgicas, que respondam à pergunta norteadora. Os filtros aplicados foram: texto completo; em idioma português, inglês, espanhol, francês e alemão; ano 
de publicação, 2007 a 2018; tipo de documento, artigo. Os critérios de exclusão definidos consistem em estudos que enfatizavam a técnica cirúrgica, ou procedimentos de nível ambulatorial que não compreendam os critérios de inclusão, revisões da literatura, relatos de experiência e estudos de caso. Os resultados da busca foram transferidos para o software Endnote X 7.2. Os artigos em duplicidade foram excluídos.

Após a busca inicial, os textos passaram por um processo de seleção por meio de três fases: primeira, a avaliação do título; segunda, a avaliação do resumo e terceira, avaliação do texto completo dos artigos que atenderam aos critérios de inclusão. Os artigos cujos títulos e resumos não apresentaram clareza para sua inclusão ou exclusão foram mantidos para a etapa seguinte de leitura integral do trabalho. Foram selecionados os artigos lidos na íntegra que se adequaram aos critérios. O processo foi realizado por dois pesquisadores independentemente. As discordâncias foram discutidas entre o par até um consenso. Os artigos selecionados foram analisados nos seguintes aspectos: o tipo de estudo; o tamanho da amostra; a definição de evento adverso cirúrgico e de complicação cirúrgica.

A síntese descritiva dos artigos selecionados foi realizada conforme suas características gerais, apresentadas no

\section{Figura 1 - Fluxograma de seleção das publicações da revisão sistemática}

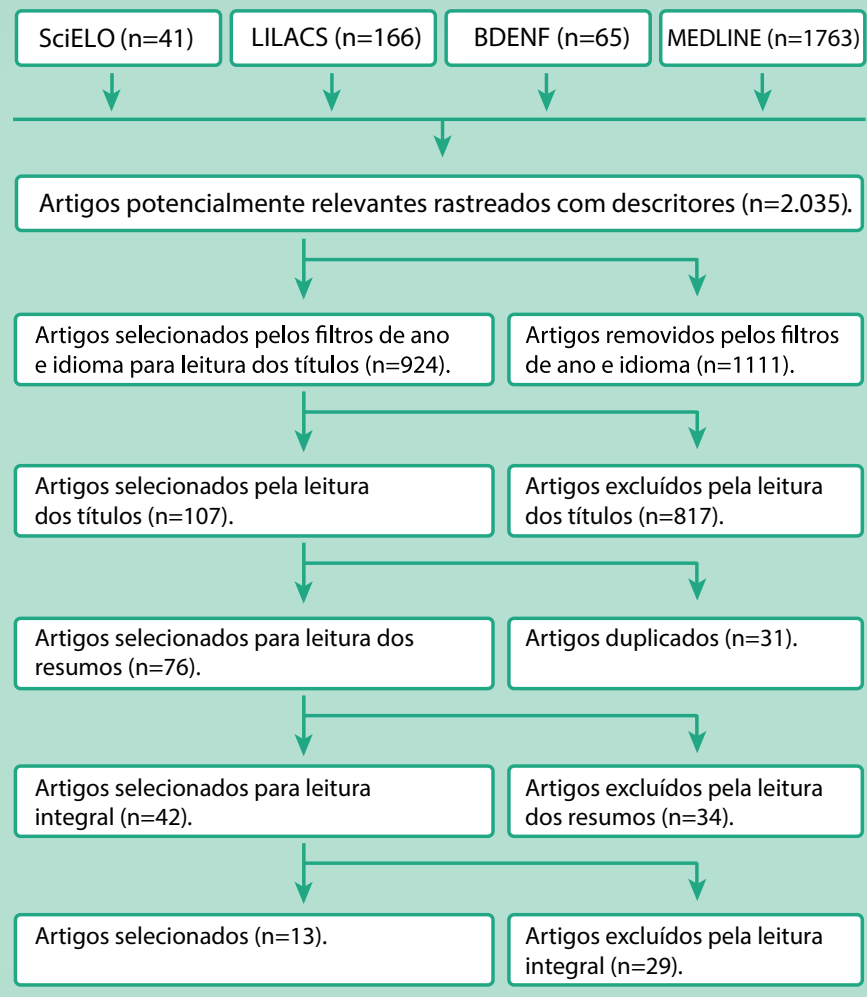

Fonte: Elaborado pelos autores

Tabela 1 - Relação de artigos obtidos por base de dados.

Descritores

Medidas de segurança AND Centro Cirúrgico.

LILACS
$\mathrm{N}^{\circ}$

31

MEDLINE
No $^{\circ}$
640

640 quadro 1. Os dados foram discutidos à luz do contexto da qualidade em saúde, com ênfase na Política de segurança do paciente.

\section{RESULTADOS}

Através da busca inicial, utilizando os referidos termos de busca, foram levantadas 2035 publicações nas bases de dados SciELO, LILACS, DBENF, MEDLINE, dessas 1111 foram desconsideradas com a aplicação dos filtros inerentes aos critérios de inclusão e exclusão do estudo. Sendo assim, 924 artigos foram submetidos ao seguinte processo de seleção: após avaliação dos títulos 817 artigos foram retirados, a avaliação dos títulos possibilitou ainda a eliminação de 31 artigos duplicados, a partir da leitura dos resumos foram removidos 34 artigos, restando 42 artigos para leitura integral e por fim 29 artigos foram excluídos por não atenderem aos quesitos.

Após a seleção, permaneceram 13 estudos que foram analisados de acordo com tipo de estudo, tamanho da amostra, definição de evento adverso cirúrgico e de complicação cirúrgica, incidência de EAs cirúrgicos e desenho da avaliação realizada. O processo de seleção e avaliação está ilustrado na Figura 1.

A maioria das publicações foi obtida por meio do cruzamento dos descritores "Segurança do paciente AND Centro cirúrgico" (34,3\%), seguido do descritor "Medidas de segurança AND Centro cirúrgico" (33,8\%) e a base de dados MEDLINE compreende a grande maioria dos artigos (1763), como se observa na Tabela 1.

Os 13 artigos selecionados apresentam os eventos adversos cirúrgicos mais frequentes. Em relação ao local de realização 


\begin{tabular}{|lccccc|}
\hline Eventos adversos* AND Centro Cirúrgico. & 32 & 10 & 16 & 5 & 3,1 \\
\hline Gestão de riscos AND Centro Cirúrgico. & 11 & 570 & 4 & 1 & 28,8 \\
\hline Segurança do paciente AND Centro Cirúrgico. & 92 & 543 & 31 & 32 & 34,3 \\
\hline Total & 166 & 1763 & 65 & 41 & $100 \%$ \\
\hline
\end{tabular}

*Eventos adversos não é um descritor do DeCS.

Fonte: Elaborado pelos autores

dos estudos, 4 foram realizados nos Estados Unidos da América e no Brasil (30,8\%), respectivamente, dois na Inglaterra $(15,3 \%)$ e um na Espanha (7,7\%), Itália(7,7\%) e Japão $(7,7 \%)$. Quanto ao ano de publicação, observa-se uma organização temporal dos estudos, com início em 2010 ininterruptamente até 2015. Em relação ao idioma principal da publicação, oito artigos foram publicados na língua inglesa $(61,5 \%)$, quatro artigos na língua portuguesa $(30,8 \%)$ e um na língua espanhola $(7,7 \%)$.
Os principais eventos adversos relatados no momento do período perioperatório está descrito no Quadro 1.

Foram identificados, eventos adversos relacionados à comunicação no serviço de saúde: falha no plano de comorbidades,

Quadro 1 - Publicações, por autor, título, país/ano, momento cirúrgico e eventos adversos.

$\begin{array}{llll}\text { Autores } & \text { Título País/Ano } & \begin{array}{l}\text { Momento } \\ \text { cirúrgico }\end{array} & \text { Eventos adversos }\end{array}$

A systematic quantitative assessment of risks associated with poor Inglaterra/2010 communication in surgical care

Wu, Rebecca L. Aufses, Jr. Arthur $\mathrm{H}$.

Characteristics and costs of surgical scheduling errors

The introduction of an acute physiological support service for surgical patients is an effective error reduction strategy

Perioperative patient safety

Takefumikitazawa. et al. indicators and Hospital surgical volumes

Prevalence and predictors of adverse eventsin older surgical patients: impact of the present on admission indicator

Hongsoo, K. et al.

Avaliação de eventos adversos

Moura, M. L. 0.

Mendes, W. Cirúrgicos em hospitais do Rio de Janeiro

Rebollo-Rodrigo, H. Madrazo-Leal, C. Gómez-Fleitas,

M.

Sistema de vigilância continua de eventos adversos los servicios quiruricos de Cantabria

Rebollo-Rodrigo, H. Madrazo-Leal, C. Gómez-Fleitas, M.

Sistema de vigilância continua de eventos adversos los servicios quiruricos de Cantabria
$\mathrm{EUA}^{* * *} / 2012$ Inglaterra/2013 Japão/2014

EUA/2010

-Falha na investigação de exame.

Pré-operatório -Ausência de tomada de decisão. -Falha no plano de comorbidades.

-Erro de lateralidade

-Procedimento incompleto

-Erro de abordagem ou abordagem indevida

-Relacionado à infusão de líquidos intravenosos

-Relacionado à medicação

-Relacionado a dispositivos

-Reação transfusão

-Corpo estranho deixado durante procedimento

-Punção/laceração acidental

-Lesão por pressão

-Embolia pulmonar ou trombose venosa profunda

-IRAS*

Pós-operatório

-Outros problemas com a ferida cirúrgica

-Infecção de ferida cirúrgica

-Infecção não relacionada à ferida cirúrgica

\section{-Complicação cirúrgica}

-IRAS

Sem momento definido

Espanha/2010
-Complicação cirúrgica

-IRAS

-Outros 


\begin{tabular}{|c|c|c|c|c|}
\hline Mattioli, G. et al. & $\begin{array}{l}\text { Near-miss events are really missed! } \\
\text { Reflections on incident reporting in } \\
\text { a department of pediatric surgery }\end{array}$ & Itália /2012 & \multirow{4}{*}{$\begin{array}{l}\text { Sem momen- } \\
\text { to definido }\end{array}$} & $\begin{array}{l}\text {-Infecção } \\
\text {-Eventos organizacionais } \\
\text {-Obstrução intestinal }\end{array}$ \\
\hline Heslin, J.M. et al. & $\begin{array}{l}\text { A } 100 \% \text { departmental mortality } \\
\text { review improves observed-to-expec- } \\
\text { ted mortality ratios and university } \\
\text { healthsystem consortium rankings }\end{array}$ & EUA/2014 & & $\begin{array}{l}\text {-Trombose venosa profunda/ embolia pulmonar. } \\
\text {-Parada respiratória } \\
\text {-Sangramento ou hematoma }\end{array}$ \\
\hline Bezerra, W. R. et al. & $\begin{array}{l}\text { Ocorrência de incidentes em um } \\
\text { centro cirúrgico: estudo documental }\end{array}$ & Brasil/2015 & & $\begin{array}{l}\text {-Cirurgia suspensa } \\
\text {-Perfuração de luva } \\
\text {-Falhas técnicas envolvendo o paciente } \\
\text {-Falhas organizacionais }\end{array}$ \\
\hline Silva, F. G. et al. & $\begin{array}{l}\text { Análise de eventos adversos em um } \\
\text { centro cirúrgico ambulatorial }\end{array}$ & Brasil/2015 & & $\begin{array}{l}\text {-Erro de processo } \\
\text {-Queixa técnica } \\
\text {-Evento adverso }\end{array}$ \\
\hline
\end{tabular}

*IRAS, Infecção relacionada à assistência: **PCR, parda cardiorrespiratória; EUA, Estados Unidos da América.

Fonte: Elaborado pelos autores

ausência de tomada de decisão, falta de investigação de exame, erro de abordagem, procedimento incompleto; eventos adversos relacionados a lesões por pressão: lesão por pressão; eventos adversos relacionados a medicamentos: erro na transfusão de líquido endovenoso e erro de medicação; eventos adversos relacionados ao uso de dispositivos para a saúde: falta de manutenção de equipamentos, erro relacionado ao dispositivo, perfuração da luva e embolia pulmonar/trombose venosa profunda e outros que consistem em eventos adversos organizacionais ou inespecíficos como: erro de processo, queixa técnica, falta de humanização no cuidado, falha técnica envolvendo paciente, falhas organizacionais, falha na previsão e provisão de materiais e eventos organizacionais.

O maior volume de eventos adversos em pacientes cirúrgicos está diretamente relacionado ao procedimento cirúrgico e ao ato anestésico: erros de lateralidade, punção/ laceração acidental, corpo estranho deixado durante o procedimento, complicações cirúrgicas, suspensão de cirurgia, alterações respiratórias/instabilidade hemodinâmica, problema relacionado ao sítio cirúrgico, obstrução intestinal, hemorragia ou hematoma pós-operatório, reação a transfusão, parada/ falha respiratória, falha na reanimação e falha na avaliação pré-operatória.

\section{DISCUSSÃO}

A busca por eventos adversos na literatura dos últimos anos teve por objetivo refletir as evidências, no que diz respeito a eventos adversos cirúrgicos. Foram analisados 13 artigos de seis países, publicados em sua maioria na língua inglesa.

No Brasil a ANVISA recebe as notificações de EA relacionadas à assistência à saúde pelo sistema Notivisa, desde fevereiro de 2014, como previsto na RDC n. 36/2013. Segundo relatório de eventos adversos, de 2014 a 2017 o número de hospitais com núcleo de segurança do paciente cadastrados nesse sistema era de 2.595, sendo que desses 969 realizaram ao menos uma notificação, a maior parte das notificações são realizadas em serviços hospitalares, sendo 126.349, o centro cirúrgico foi considerado o quarto setor que mais notifica eventos adversos no hospital com 4.873 notificações ${ }^{14}$. A ideia de ter dados referentes a eventos adversos de todo o país compilados em um único lugar é boa e necessária, contudo o sistema sofre uma subnotificação severa, tornando os dados em parte obsoletos ${ }^{7}$.

Complicações cirúrgicas têm potencial para causar grandes danos. A incidência de pacientes com eventos adversos cirúrgicos é de 3,5\%, de todos os pacientes internados e a proporção de pacientes cirúrgicos com eventos adversos é de $5,9 \%{ }^{15}$. Um estudo realiza- do, no Rio de Janeiro, sobre as características de eventos adversos evitáveis observou predominância de EAs evitáveis nas enfermarias com $56,9 \%$ e no centro cirúrgico em $30,8 \%$ dos casos, o mesmo estudo buscou os fatores contribuintes para a ocorrência de EAs evitáveis e identificou a não adesão a normas, ou seja, em 55,9\% dos casos os profissionais não seguem os protocolos ou diretrizes clínicas ${ }^{16}$.

Carvalho e Vieira ${ }^{17}$ afirmam que o profissional de saúde tem que aceitar que é falível, pois erros acontecem e vários fatores externos e internos contribuem para o erro. Os eventos adversos resultantes de erros técnicos ou abordagens inadequadas acarretam em sérios danos, que vão da incapacidade temporária ao óbito, contudo os danos podem ser minimizados com planos de ação e trabalho em equipe.

O protocolo de segurança cirúrgica da OMS tem a proposta de aplicar um checklist cirúrgico que envolve toda equipe cirúrgica e pode ser adaptado para qualquer realidade institucional, ele compreende três momentos cruciais para realizar a checagem o sing in - antes da indução anestésica, time - out- antes da incisão cirúrgica e o sing out- antes do paciente sair da sala de cirurgia. Cada um desses momentos é composto por um conjunto de itens e procedimentos a serem checados antes de partir para o próximo momento, o protocolo propõe ainda o monitoramento de alguns indicadores ine- 
rentes à segurança cirúrgica, ao percentual de pacientes que recebeu antibiótico-profilaxia no momento adequado, ao número de cirurgias em local errado, ao número de cirurgias em paciente errado, ao número de procedimentos errados, à taxa de mortalidade cirúrgica intra-hospitalar ajustada ao risco e à taxa de adesão ao checklist, que permitem a identificação de pontos críticos na assistência cirúrgica para desenvolvimento de ações específicas e efetivas ${ }^{3,18-20}$.

Pacientes cirúrgicos, assim como outros pacientes hospitalizados, estão sujeitos as infecções relacionadas à assistência, como: sepse pós-operatória, infecção sítio cirúrgico, infecção não relacionada ao sítio cirúrgico e outras infecções. O primeiro desafio global para segurança do paciente, lançado em 2005, teve o intuito de promover a higiene das mãos entre profissionais e cidadãos por meio do protocolo de higiene das mãos, reduzindo e prevenindo as infecções relacionadas à assistência à saúde ${ }^{10,11}$.

Padovezel $^{22}$ alerta sobre as IRAS serem um problema de saúde pública e diz que, mesmo com algumas iniciativas, as proporções do problema são ocasionalmente subestimadas, obtendo suporte para ações de prevenção insuficientes.

A autonomia do paciente e a comunicação médico/paciente é crucial ao considerar o princípio da capacitação no cuidado centrado na pessoa, pois auxilia o paciente a desenvolver os conhecimentos, as aptidões e a confiança necessária para gerir as informações sobre si e tomar decisões embasadas sobre sua própria saúde assumindo o protagonismo de seu cuidado ${ }^{10,12}$.

Em geral, os eventos adversos mais frequentes no centro cirúrgico são complexos por se tratarem de pacientes críticos, no entanto, são passíveis de ser prevenidos por meio da sistematização da assistência, da educação permanente da equipe multiprofissional, da aplicação efetiva de protocolos de prevenção de trombose, do checklist de segurança cirúrgica e do cumprimento de diretrizes e protocolos baseados em evidência ${ }^{23}$. Um estudo realizado em três hospitais brasileiros em 2013, identificou fatores contribuintes para ocorrência de eventos adversos e observou que em 55,9\% dos casos o fator contribuinte estava relacionado ao não cumprimento de protocolos ou diretrizes clínicas ${ }^{16}$.

A partir desse estudo, os profissionais de saúde podem rever os processos e seus fluxos dentro do bloco cirúrgico, a fim de desenvolver ou fortalecer barreiras para reduzir os danos e contribuir para segurança do paciente cirúrgico.

\section{CONCLUSÃO}

A partir deste estudo, os profissionais de saúde podem rever os processos e seus fluxos dentro do bloco cirúrgico a fim de desenvolver ou fortalecer barreiras para reduzir os danos e contribuir para segurança do paciente cirúrgico.

O estudo teve como limitação a inexistência de uma classificação mundial dos eventos adversos, com nomenclatura própria que auxilie a identificação dos eventos adversos em diferentes países.

\section{Referências}

1. Penna MM, Duarte I,Cohen C, Oliveira RA. Concepções sobre o princípio da não maleficência e suas relações com a prudência. RevBioét(impr). 2012; 20(1): 78-86.

2. Haynes AB, Weiser TG, Bery WR, Lipsitz SR, BreizatAHS, Dellinger EP. A surgical safety checklist to reduce morbidity and mortality in a global population. N Engl J Med. 2009; 360(1): 491- 499.

3. Organização Mundial da Saúde - OMS. Segundo desafio global para a segurança do paciente: cirurgias seguras salvam vidas (Orientações para cirurgia segura da OMS). Rio de Janeiro: Organização Pan-Americana da Saúde, Ministério da Saúde, Agência Nacional de Vigilância Sanitária; 2009.

4. Brasil. Anexo 03: Protocolo para Cinurgia Segura. [Internet]. Ministério da Saúde/ Anvisa/ Fiocruz; jul.2013a [Acesso em: 31 de agosto de 2018]. Disponível em: https://uww20.anvisa.gov.br/segurancadopaciente/index.php/publicacoes/it em/protocolo-de-cinurgia-segura. 5. Brennan TA, Leape $L L$, Laird NM, Hebert L, Localio AR, Lawthers AG, et al. Incidence of adverse events and negligence in hospitalized patients: results of the Harvard Medical Practice Study I. N Engl J Med. 1991; 324(6): 370-376.

6. Kohn LT, Corrigan JM, Donaldson MS. To err is human: building a safer health system. Washington, DC: National Academy Press; 2000.

7. Vila Nova VR, Marques JL, Santos TB, Oliveira JR, Prates DO, Santos AS, et al. Analysis of the patient safety culture in a public hospital in brazil", Intemational Journal of Development Research. 2019; 9(3): 26254-26259.

8. Brasil. Ministério da Saúde (BR). Portaria $n^{\circ} 529$, de $1^{\circ}$ de abril de 2013. Institui o Programa Nacional de Segurança do Paciente (PNSP). Diário Oficial da União; 2013b; (62):43. 9. Paiva MCMS, Paiva SAR, Berti HW. Eventos Adversos: análise de um instrumento de notificação utilizado no gerenciamento de enfermagem. RevEscEnferm USP. 2010; 44 (1):287-94. 10. Fiocruz. Simplificando o cuidado centrado na pessoa: o que todos devem saber sobre o cuidado centrado na pessoa - Guia rápido. Fiocruz: Instituto de Comunicação Científica e Tecnológica em Saúde; 2016.

11. Cassiani, SHB. A segurança do paciente e o paradoxo no uso de medicamentos. Rev.
Bras. Enferm. 2005; 58(1): 95-99.

12. IBSP. Termo de Consentimento: como elaborar e aplicar para evitar problemas jurídicos. IBSP- Instituto Brasileiro para Segurança do Paciente. Cultura de Segurança \& Gestão. São Paulo/SP: 2018.

13. Weiser TG, Regenbogen SE, Thompson KD, Haynes AB, Lipsitz SR, Berry WR, et al. An estimation of the global volume of surgery: a modeling strategy based on available data. Lancet. 2008; 372(9633): 139-44.

14. ANVISA. Relatórios dos Estados: eventos Adversos [Acesso 17 Out 2017]. 2017. Disponivel em: https://uww20.anvisa.gov.br/segurancadopaciente/index.php/publicacoes/ category/relatorios-dos-estados.

15. Moura MLO, MendesW. Avaliação de eventos adversos cirúrgicos em hospitais do Rio de Janeiro. RevBrasEpidemiol. 2012; 15(3): 523-35.

16. Mendes W, Pavão ALB, Martins M, Moura MLO, Travassos C. Características de eventos adversos evitáveis em hospitais do Rio de Janeiro. Rev.Assoc. Med. Bras. 2013; 9(5): 421-428. 17. Carvalho M, Vieira AA. Erro médico em pacientes hospitalizados. J Pediatr. 2002; 78 (4): 261-8.

18.Camanho G. Cirurgia segura para todos. Rev. Bras. Ortop. 2014; 49(6): 553-554. 19. WHO. World Alliance For Patient Safety. Implementation manual - surgical safety checklist - safe surgery saves lives. [Intemet]. Geneva:WHO; 2008 [Acessoem: 23 de maio de 2017]. Disponivelem: http://uww.who.int/patientsafety/safesurgery/ss_decklist/en/index.html. 20. CatchpoleK, Russ S. The problem with checklists. Bmj Quality \& Safety.2015;24(9):545-549. 21.Brasil.Assistência Segura: uma reflexão teórica aplicada àpratica.Série segurança do paciente e qualidade em serviços de saúde. Ministério da Saúde/ Anvisa/ Fiocruz; 2017.

22. Padovezei MC, Fortaleza CMCB. Infecções relacionadas à assistência à saúde: desafios para a saúde pública no Brasil. ver. Saúde Pública. 2014; 48(6): 995-1001.

23. Costa Junior AS. Assessment of operative times of multiple surgical specialties in a public university hospital. Einstein (São Paulo). 2017; 15(2): 200-205. 